\title{
Evolving Challenges in Diagnosis of Renal Vasculitis
}

\author{
Than Aung, ${ }^{\mathrm{a}, \mathrm{b}}$, Haresh Tulsidas ${ }^{\mathrm{a}}$
}

\begin{abstract}
Renal-limited vasculitis is a rare anti-neutrophil cytoplasmic antibody (ANCA)-associated vasculitis that presents only with a renal manifestation in the absence of other organs involvement. In this report, a 50-year-old female presented with nonspecific symptoms and anemia, who was subsequently discovered to have renal-limited vasculitis. After receiving a combination of steroid and immunosuppressive therapy, she recovered uneventfully without further relapse. A wide range of nonspecific presenting symptoms and the insidious nature of renal disease often delay in early recognition of renal-limited vasculitis. Keeping a lower threshold of initiating vasculitis workup helps detect the earlier diagnosis which is crucial in management with improved renal outcome.
\end{abstract}

Keywords: Antineutrophil cytoplasmic antibody-associated vasculitis; Renal-limited vasculitis; Immunosuppressive therapy

\section{Introduction}

Diagnosis of anti-neutrophil cytoplasmic antibody (ANCA)-associated vasculitis is often easily missed and delayed because the condition generally presents with a variety of nonspecific symptoms. We report a case who presented with symptomatic anemia eventually was found to have renal-limited vasculitis (RLV).

\section{Case Report}

A 50-year-old female, with past medical history of hypertension and dyslipidemia, visited our outpatient clinic for experiencing reduced effort tolerance, and weight loss of $3 \mathrm{~kg}$ from 37 to 34 over last 2 months. She noted her activity became slower in doing house chores and climbing a flight of stairs.

She denied other systemic symptoms such as cough, hem-

Manuscript submitted January 10, 2021, accepted January 23, 2021

Published online February 8, 2021

aDepartment of Internal Medicine, Singapore General Hospital, Outram Road, Singapore 169608, Singapore

${ }^{b}$ Corresponding Author: Than Aung, Department of Internal Medicine, Singapore General Hospital, Outram Road, Singapore 169608, Singapore.

Email: than.aung@singhealth.com.sg

doi: https://doi.org/10.14740/jmc3648 optysis and night sweats. Examination was unremarkable including breast and lymph nodes examination.

Laboratory tests found normocytic anemia with hemoglobin $9.8 \mathrm{~g} / \mathrm{dL}(12$ - $16 \mathrm{~g} / \mathrm{dL})$, albumin $30 \mathrm{~g} / \mathrm{L}(40-51 \mathrm{~g} / \mathrm{L})$ and the rest of renal function, thyroid function and liver function tests were normal.

Further study on anemia disclosed an iron deficiency anemia as transferrin saturation of 7.1\% (12-50\%), ferritin of 241 $\mu \mathrm{g} / \mathrm{L}(18-339 \mu \mathrm{g} / \mathrm{L})$, normal vitamin B12 and serum folate. Computed tomography (CT) scan of chest, abdomen and pelvis for evaluation of her loss of weight revealed nonspecific bronchial wall thickening with mucous plugging of airways in the right middle lobe and lingular lobe suggesting airway inflammation. No enlarged lymph nodes were visible. Subsequent sputum tests for acid fast bacilli (AFB) smear and nucleic acid amplification test for tuberculosis showed no evidence of mycobacteria infection.

A month later, on reviewing her symptoms, she experienced a few episodes of intermittent low-grade fever of $38^{\circ} \mathrm{C}$ in the morning. She was not aware of any symptoms of joint pain, muscle ache, skin rash, alopecia, mouth ulcers or Raynaud's phenomenon. No significant finding was detected on examination.

Hemoglobin level decreased from $9.8 \mathrm{~g} / \mathrm{dL}$ to $7.8 \mathrm{~g} / \mathrm{dL}$. White blood cell, platelet and differential cell counts were normal. The additional laboratory values were as follows: normal renal panel and liver function test except serum albumin 25 $\mathrm{g} / \mathrm{L}$, procalcitonin $0.3 \mu \mathrm{g} / \mathrm{L}(<0.50 \mu \mathrm{g} / \mathrm{L})$, erythrocyte sedimentation rate (ESR) $33 \mathrm{~mm} / \mathrm{h}(3-20 \mathrm{~mm} / \mathrm{h})$ and C-reactive protein (CRP) $111 \mathrm{mg} / \mathrm{L}(0.2-9.1 \mathrm{mg} / \mathrm{L})$. Urinalysis was unremarkable. Urine protein creatinine ratio was $0.24 \mathrm{~g} / \mathrm{g}(<0.20$ $\mathrm{g} / \mathrm{g})$. Chest X-ray showed no significant abnormality. There was no bacterial growth on blood cultures.

Gradual declining hemoglobin in conjunction with loss of weight prompted her for endoscopic evaluation to explore occult bleeding or gastrointestinal malignancy. Esophagogastroduodenoscopy and colonoscopy findings were normal. She continued to have low-grade fever. In view of the CT finding of airway inflammation, she underwent bronchoscopy. Apart from finding of small amount of mucus secretion in the right middle lobe and the left lingula, no endobronchial lesion was documented. Bronchoalveolar lavage fluid was tested for tuberculosis. AFB smear test, nucleic acid amplification test and culture all turned out negative for mycobacteria. There was normal left ventricular ejection fraction of 58\% with no evidence of infective endocarditis on echocardiography.

Although the patient underwent extensive investigations including CT scan and endoscopic evaluations which excluded infections and malignancy, she remained not feeling herself. Her persistent unexplained anemia and intermittent low-grade 
fever aroused us a suspicion of an alternative diagnosis, autoimmune disease.

Repeated urine microscopy showed red cell 28 per high power field ( 0 - 3 cell), white cell 13 per high power field ( 0 - 5 cell). Urine protein creatinine ratio slightly rose up to $0.6 \mathrm{~g} / \mathrm{g}$. Her serum creatinine increased from 79 to $90 \mu \mathrm{mol} / \mathrm{L}$ (37 - 75 $\mu \mathrm{mol} / \mathrm{L})$. Further study with urine phase contrast examination exposed dysmorphic red cells of 18 .

Antinuclear antibody, double-stranded DNA antibody, extractable nuclear antigen (ENA) antibodies panel (anti-Ro, anti-La, anti-ribonucleoprotein, anti-smith antibody, anti-Jo antibody) tests were negative.

Later ANCA profile came out with an elevated level of anti-myeloperoxidase (MPO) antibody $124 \mathrm{RU} / \mathrm{mL}(<20 \mathrm{RU} /$ $\mathrm{mL}$ ) and normal level of anti-proteinase 3 (PR3) antibody. Anti-glomerular basement antibody was negative.

She was highly suspicious of having ANCA-associated vasculitis with renal involvement. Subsequent renal biopsy reported pauci-immune glomerulonephritis with necrotizing arteritis, $11 \%$ of crescent granuloma which confirmed the diagnosis of MPO-positive ANCA-associated vasculitis with primarily renal involvement.

She was treated with glucocorticoid and rituximab therapy. Her feeling of tiredness and low-grade fever subsided. After 1 month, her hemoglobin level was back to normal range $(12.8 \mathrm{~g} / \mathrm{dL})$ and serum creatinine remained plateau at $92 \mu \mathrm{g} / \mathrm{L}$. Her MPO level gradually decreased to $24 \mathrm{RU} / \mathrm{mL}$ then to 11 $\mathrm{RU} / \mathrm{mL}$, and subsequently to a normal range in the following months. She had no relapse on follow-up visits.

\section{Discussion}

RLV is a type of ANCA-associated vasculitis, which affects the kidney but no other organs, characterized by necrotizing inflammation of small vessels, the relative paucity of immune deposits and an association with circulating ANCA. ANCAassociated vasculitis includes granulomatosis with polyangiitis (GPA), microscopic polyangiitis (MPA), RLV, and eosinophilic granulomatosis with polyangiitis (EGPA, Churg-Strauss). They share similar features of focal necrotizing and crescentic glomerulonephritis with few or no immune complex deposition on renal histology [1].

Incidence of pauci-immune renal-limited glomerulonephritis in the USA has been found to be around 3 per million per year with a rate being observed higher in white people [2].

It can occur at any age, any racial group with no gender predominance.

The etiology of ANCA-associated vasculitis has not been clearly understood. It is speculated to be an autoimmune disease with the presence of ANCA in most cases. Genetic factors are likely to play a role in association with ANCA-associated vasculitis [3]. Disease pathogenesis may be linked to an environmental factor such as exposure to silica, infectious agents like Staphylococcus aureus and exposure to certain drugs namely propylthiouracil [4]. ANCA-associated vasculitis has a predilection for the kidney. The morphological distinctive characteristic of ANCA-associated vasculitis is a necrotizing crescentic glomerulonephritis with scanty immune deposits.

Diagnosing vasculitis can be challenging because nonspecific clinical presentation mimics infection or malignancy. Infection and malignancy should be excluded first. Patients with mycobacteria infections generally present in a similar indolent course with low-grade fever, malaise, weight loss with nonspecific physical findings. Vasculitis may present and progress slowly over months or rapidly over days. Patients may report several weeks to months of constitutional symptoms before the appearance of focal disease manifestations particularly rash, arthritis, lung, or renal involvement. Additionally, focal signs of vasculitis may be short-lived reflections, for example, transient arthritis, purpuric rash or skin ulceration, and mouth ulceration. In RLV the insidious nature of renal disease which usually presents late with advanced renal failure and symptoms of uremia, contributes to delay diagnosis [5]. General laboratory findings of normochromic normocytic anemia, hypoalbuminemia and elevation of systemic inflammatory markers are nonspecific.

Normocytic anemia is a complication of ANCA-associated renal vasculitis. Most of the patients had renal anemia and some had anemia of chronic disease. The causes of anemia were multifactorial and hepcidin-induced changes in iron metabolism appeared to be one of the mechanisms of anemia.

Unexplained anemia associated with renal and nonspecific pulmonary involvement in patients with prolonged illness should prompt the physician to suspect a rare systemic disorder. Vasculitis should be an alternative consideration.

In our case, the manifestation of nonspecific lethargy, persistent anemia followed by low-grade fever led us to suspect a mycobacterial infection and the subsequent appearance of microscopic hematuria rectified us the way to diagnose a vasculitis.

Urinalysis is an invaluable simple test to determine the presence of hematuria that can provide a clue to early renal involvement.

Vasculitis should be suspected in any patient with hematuria and proteinuria. The presence of extra-renal vasculitis features particularly naso-sinus and ear disease, hemoptysis with radiological infiltrates and pulmonary nodules should arouse clinicians' lower thresholds for initiating a vasculitis workup.

For patients with a high pretest probability of disease, the positive ANCA testing can timely detect the renal vasculitis.

Although there were no apparent focal signs of vasculitis, our patient was diagnosed with ANCA-associated vasculitis in a relatively early course of the disease with near-normal serum creatinine level by renal biopsy which was prompted by positive MPO ANCA.

Diagnosis of vasculitis depends on the triad of clinical features, serology and histology.

The typical renal biopsy in most patients shows glomerulonephritis associated with few or no immune deposits in glomeruli, a pauci-immune necrotizing glomerulonephritis with crescent formation.

Treatment of ANCA-associated vasculitis consists of the initial remission induction therapy to control the disease activity followed by the remission maintenance therapy to prevent 
disease relapse.

Glucocorticoid with immunosuppressive therapy either rituximab or cyclophosphamide are indicated for the patients with active glomerulonephritis to induce remission. For induction of remission, a single course of rituximab therapy was non-inferior to cyclophosphamide-based regimen in renal outcome and safety in patients with ANCA-associated vasculitis. There was no difference in adverse events [6].

Maintenance therapy using therapeutic alternatives that have reduced toxicity is usually given for 12 - 24 months after remission is achieved.

The patients with positive MPO ANCA without respiratory tract involvement who become MPO-ANCA negative at the end of induction therapy can be considered as a low-risk group of relapses like our patient. These patients may be followed by careful monitoring without maintenance therapy $[7,8]$.

The prognosis of a patient depends on the severity of renal disease at the time of diagnosis and its response to treatment. Although remission can be achieved by immunosuppressive therapy, the active disease and treatment-related complications remain the main causes of mortality to the majority of patients with ANCA-associated vasculitis [9].

\section{Learning points}

Uncommonness and nonspecific presentation of ANCA-associated vasculitis give rise to a challenging clinical case for diagnosis and result in delay in treatment. Our case highlights the importance of keeping a high index of suspicion of vasculitis in the patient experiencing nonspecific symptoms, anemia, weight loss with an evolving episode of microscopic hematuria, who was subsequently diagnosed as an ANCA-associated renal vasculitis.

\section{Acknowledgments}

I would like to thank the Department of Internal Medicine for assisting me through the process of this case study.

\section{Financial Disclosure}

None to declare.

\section{Conflict of Interest}

None to declare.

\section{Informed Consent}

A verbal consent was obtained.

\section{Author Contributions}

Than Aung: conception and design of article; acquisition, analysis and interpretation of data; drafting the manuscript. Haresh Tulsidas: revising the manuscript critically for important intellectual content.

\section{Data Availability}

The authors declare that data supporting the findings of this study are available within the article.

\section{References}

1. Jennette JC, Falk RJ, Andrassy K, Bacon PA, Churg J, Gross WL, Hagen EC, et al. Nomenclature of systemic vasculitides. Proposal of an international consensus conference. Arthritis Rheum. 1994;37(2):187-192.

2. Lionaki S. The prevalence of Pauci-immune glomerulonephritis in western countries. Kidney Dis. 2015.

3. Jagiello P, Gross WL, Epplen JT. Complex genetics of Wegener granulomatosis. Autoimmun Rev. 2005;4(1):42-47.

4. de Lind van Wijngaarden RA, van Rijn L, Hagen EC, Watts RA, Gregorini G, Tervaert JW, Mahr AD, et al. Hypotheses on the etiology of antineutrophil cytoplasmic autoantibody associated vasculitis: the cause is hidden, but the result is known. Clin J Am Soc Nephrol. 2008;3(1):237-252.

5. David Jayne. Oxford clinical nephrology series. Rheumatology and the Kidney. 2012.

6. Geetha D, Specks U, Stone JH, Merkel PA, Seo P, Spiera $\mathrm{R}$, Langford CA, et al. Rituximab versus cyclophosphamide for ANCA-associated vasculitis with renal involvement. J Am Soc Nephrol. 2015;26(4):976-985.

7. Hogan SL. Understanding long term remission off therapy in ANCA associated vasculitis. Kidney Int Rep. 2019.

8. Gapud EJ, Manno R, Seo P, Hanouneh M, Geetha D. Long-term clinical course of antineutrophil cytoplasmic antibody-associated vasculitis patients off maintenance therapy. Cureus. 2018;10(3):e2372.

9. Little MA, Nightingale P, Verburgh CA, Hauser T, De Groot K, Savage C, Jayne D, et al. Early mortality in systemic vasculitis: relative contribution of adverse events and active vasculitis. Ann Rheum Dis. 2010;69(6):1036-1043. 